\title{
AN ANALYSIS OF STEADY-STATE DISTRIBUTION IN $M / M / 1$ QUEUEING SYSTEM WITH BALKING BASED ON CONCEPT OF STATISTICAL MECHANICS
}

\author{
IkUo Arizono ${ }^{1, *}$ And Yasuhiko TAKemoto ${ }^{2}$
}

\begin{abstract}
The phenomenon of balking has been considered frequently in the steady-state analysis of the $M / M / 1$ queueing system. Balking means the phenomenon that a customer who arrives at a queueing system leaves without joining a queue, since he/she is disgusted with the waiting queue length at the moment of his/her arrival. In the traditional studies for the steady-state analysis of the $M / M / 1$ queueing system with balking, it has been typically assumed that the arrival rates obey an inverse proportional function for the waiting queue length. In this study, based on the concept of the statistical mechanics, we have a challenge to extend the traditional steady-state analysis model for the $M / M / 1$ queueing system with balking. As the result, we have defined an extended analysis model for the $M / M / 1$ queueing system under the consideration of the change in the directivity strength of balking. In addition, the procedure for estimating the strength of balking in this analysis model using the observed data in the $M / M / 1$ queueing system has been also constructed.
\end{abstract}

Mathematics Subject Classification. 60K25, 90B22, 82B31.

Received October 31, 2018. Accepted May 21, 2019.

\section{INTRODUCTION}

Congestion occurs in various fields and yields economic and mental loss. Chen et al. [5] have discussed the highway congestion in USA and reported the utility of the ramp metering control policy. In Disneyland, street performers amuse customers waiting in queue in order to alleviate their annoyance. Also, the fast pass system in Disneyland is very famous as a strategy for minimizing the negative aspect for waiting. The fast pass system allows customers to participate in other activities while they stand in a queue at their desired activity in an appointed time. Note that this queue can be interpreted as a virtual queue. In this mean, all service and hospitality experiences require eventually customers to wait at some point in the service process [8]. Hence, many researchers and practitioners have discussed and investigated the congestion and queue to minimize the negative aspects of waiting $[21,27,30,32,34]$.

The long waiting time annoys customers waiting in queue. Also, a customer who arrives at a queue may hesitate to join the queue due to the long waiting queue length at the moment of his/her arrival. There are

Keywords. $M / M / 1$ queueing system, balking, statistical mechanics, entropy, potential energy, steady-state analysis.

1 Okayama University, 3-1-1 Tsushima-naka, Okayama 700-8530, Japan.

2 Kindai University, 3-4-1 Kowakae, Higashi-Osaka, Osaka 577-8502, Japan.

*Corresponding author: arizono@okayama-u.ac.jp 
some customers leaving without joining the queue as the result. This phenomenon is called balking. Generally, balking means the phenomenon that a customer who arrives at a queueing system leaves without joining a queue, since he/she is disgusted with the waiting queue length at the moment of his/her arrival.

In the queueing theory for analyzing congestion phenomena of systems, balking in queueing system has been considered frequently $[10,15,18,22,23,31,38]$. Then, balking is treated as stochastic behavior in queueing systems. The steady-state analysis of queueing systems under the consideration of balking has been discussed continually based on the theory of stochastic processes. For example, a variety of Markovian queueing systems with balking have been considered [1,9,17,24-26,35]. On the other hand, Cruz et al. [6] and Cruz and Smith [7] have investigated a state-dependent $\mathrm{M} / \mathrm{G} / \mathrm{c} / \mathrm{c}$ queueing networks in which the service rate is subject to congestion. Further, Hassin and Haviv [13] and Hassin [14] have written a comprehensive literature review regarding balking in $M / M / 1$ queues with strategic customers, respectively. Among them, in the traditional studies by Abou-ElAta and Hariri [1], El-Sherbiny [9], Natvig [25], and Sztrik [35], it has been typically supposed that the arrival rates obey an inverse proportional function for the waiting queue length.

Suppose the $M / M / 1$ queueing system described on the service rate $\mu$ and the arrival rate $\lambda$. In addition, $\rho=$ $\lambda / \mu$ is known as traffic intensity. Then, the service rate $\mu$ and the arrival rate $\lambda$ are given as constants respectively regardless of the waiting queue length in the $M / M / 1$ queueing system without balking. The relationship of $\rho=\lambda / \mu<1$ presents the prerequisite condition in order that the steady-state probability distribution exists in the $M / M / 1$ queueing system without balking. On the other hand, since a customer who has arrived at the system leave without joining the queue depending on the waiting queue length in the $M / M / 1$ queueing system with balking, the relationship of $\rho=\lambda / \mu<1$ is not necessarily required for the existence of the steady-state probability distribution in the $M / M / 1$ queueing system with balking. Hereafter, the term "arrival" is used in a meaning of the actual arrival that the customer joins the queue in the $M / M / 1$ queueing system. The arrival rate at the waiting queue length $n(n \geq 0)$ is represented as $\lambda_{n}$. Remark that $\lambda_{0}=\lambda$, since it is trivial that balking for the waiting queue length does not occur when $n=0$. Further, it is naturally assumed that the relationship of $\lambda_{n} \geq \lambda_{n+1}$ for any $n$ is satisfied in the $M / M / 1$ queueing system with balking. Further, we describe the steady-state probability for the queueing length $n$ as $P_{n}$.

Based on the above notations, under the traditional steady-state analysis for the $M / M / 1$ queueing system with balking, the system of equations about the steady-state probabilities can be presented as follows:

$$
\begin{aligned}
\lambda_{0} P_{0}-\mu P_{1}=\lambda P_{0}-\mu P_{1}=0 \quad(n=0), \\
\lambda_{n-1} P_{n-1}-\left(\lambda_{n}+\mu_{n}\right) P_{n}+\mu_{n+1} P_{n+1}=0 \quad(n \geq 1) .
\end{aligned}
$$

From the relationship of equations (1.1) and (1.2), we can derive

$$
P_{n}=\frac{\prod_{k=0}^{n-1} \lambda_{k}}{\mu^{n}} P_{0} .
$$

In the traditional studies with consideration of balking, it has been generally assumed that all of the arrival rate $\lambda_{n}$ are known. Under the assumption that all of $\lambda_{n}$ are known, we can evaluate the steady-state probability $P_{n}$ of the $M / M / 1$ queueing system with balking by using equation (1.3). Especially, in the case without balking, since the arrival rate for any $n$ is constant as $\lambda_{n}=\lambda$, we derived the following conventional relationship by using equation (1.3):

$$
P_{n}=(1-\rho) \rho^{n} .
$$

On the other hand, in the traditional studies by Abou-El-Ata and Hariri [1], El-Sherbiny [9], Natvig [25], and Sztrik [35], the arrival rate $\lambda_{n}$ has been typically assumed as follows:

$$
\lambda_{n}=\frac{\lambda}{n+1} .
$$


Then, based on equations (1.3) and (1.5), the steady-state probability $P_{n}$ in the $M / M / 1$ queueing system with balking is obtained as

$$
P_{n}=\frac{\rho^{n} e^{-\rho}}{n !}
$$

Equation (1.6) is well-known as the probability function of the Poisson distribution. Equation (1.5) has been employed frequently without justifiable reason. We understand that the usage of equation (1.5) is due to the mathematically preferable conclusion that the Poisson distribution form is derived. However, it does not necessarily guarantee that the arrival rate by equation (1.5) is theoretically justified. At least, the decline of arrival rates by balking is probably diverse according to the relation between the waiting queue length and the degree of occurrence of balking. Furthermore, depending on the queueing system, the relation between the waiting queue length and the degree of occurrence of balking may be different. In some queueing systems, the arrival of customers decreases sharply with a slight increase in waiting queue length. In contrast, the arrival of customers does not decrease much as the waiting queue length increases, and then there are queueing systems that a long queue is formed in consequence. Therefore, in the case of analyzing the queueing system with balking, the relation between the waiting queue length and the degree of occurrence of balking should be considered. In this study, let's call the relation between the waiting queue length and the degree of occurrence of balking by the "directivity strength" of balking. However, the diversity of the directivity strength of balking has not been considered in equations (1.5) and (1.6). In practice, it is not so easy to estimate the respective arrival rates $\lambda_{n}, \forall n$ through observing the queueing system in actual.

By the way, as one of analysis models to derive the steady-state probability distribution of the queueing system without balking, Guiasu [12] has suggested a model based on the maximum entropy principle in the information theory separately from conventional stochastic analysis. Further, Arizono et al. [2] have proposed an entropy model for analyzing the steady-state probability distribution of the $M / M / c$ queueing system. Furthermore, there are many studies about queueing systems associated with the concept of the entropy. See Borzadaran [3], Jain and Dhakad [16], Kouvatsos and Tabet-Aouel [19], Kouvatsos et al. [20], Prabhakar [28], Singh and Tiwari [33], and Wang et al. [37]. However, note that balking has not been considered in the above studies based on the concept of the entropy.

The concept of the statistical mechanics [4] including the concept of the entropy has been proposed. In this paper, we address an extended analysis of the steady-state probability distribution of the $M / M / 1$ queueing system under the consideration of balking based on the concept of the statistical mechanics. In such an extension, the adjustment by the directivity strength of balking is considered based on the concept of the statistical mechanics. Incidentally, the key role of the statistical mechanics is to predict the measurable properties of a macroscopic system based on the properties and behaviour of the microscopic components of the system. Statistical mechanics deals with the laws of probability so that it does not strictly concentrate on the behaviour of every individual particle at the micro-level and therefore it is suitable for the approximate analysis of complex systems [36].

As mentioned previously, in the traditional analysis of the $M / M / 1$ queueing system with balking, the steadystate probability distribution has been derived under the typical assumption that the arrival rates obeys an inverse proportional function for the waiting queue length in equation (1.5). By contrast, note that, in this study, through analyzing the model constructed based on the concept of the statistical mechanics, a formula for deriving arrival rates for respective waiting queue lengths can be derived. In this case, the formula of arrival rates for respective waiting queue length is established under considering the adjustment of the the directivity strength of balking. Furthermore, by using this formula, we can estimate arrival rates for respective waiting queue lengths based on some observed data about the frequency of the waiting queue lengths in the $M / M / 1$ queueing system.

In Section 2, we address a model for analyzing the $M / M / 1$ queueing system under the consideration of balking. In concrete, we construct a novel analysis model for deriving the steady-state probability distribution of the $M / M / 1$ queueing system with balking based on the concept of the statistical mechanics. This novel 
analysis model is called the extended analysis model. Section 3 indicates some numerical results based on the extended analysis model considered in Section 2. Based on the extended analysis model, the steady-state probability distribution and the arrival rates in each waiting queue length of the $M / M / 1$ queueing system with balking are formulated in theory. Further, in Section 4, by utilizing the extended analysis model, the procedure for estimating the directivity strength of balking through the observation result about the frequency of the waiting queue lengths in the $M / M / 1$ queueing system with balking is also constructed. Some numerical results through the Monte Carlo simulation are shown. Finally, Section 5 concludes this paper.

\section{Proposal of ANAlysis model BASED on CONCEPt OF STATistical MeChanics}

In this section, a novel analysis model for deriving the steady-state probability distribution of the $M / M / 1$ queueing system with balking is constructed based on the concept of the statistical mechanics. Through analyzing the model constructed based on the concept of the statistical mechanics, a formula for deriving arrival rates for respective waiting queue lengths can be derived. This analysis model constructed based on the concept of the statistical mechanics is called the extended analysis model. Further, by utilizing this formula, the procedure for estimating the arrival rates through some observed data about the frequency of the waiting queue lengths in the $M / M / 1$ queueing system with balking is also investigated.

In the physics research field, particularly a thermodynamic research field, there is a concept of the statistical mechanics for evaluating the state of systems [4]. The concept of the statistical mechanics consists of both concepts of the entropy and the potential energy. The entropy is interpreted as a criterion for evaluating irregularity of systems. And, the potential energy is interpreted as a criterion for evaluating instability of systems. Then, in the statistical mechanics, a state of the system is converged according to the balance between the entropy and the potential energy of the system [4]. Then, under the concept of the statistical mechanics, we consider the steady-state probability distribution of the $M / M / 1$ queueing system with balking by the relationship of the entropy and the potential energy.

Define the state probability vector $\vec{P}$ as $\vec{P}=\left(P_{0}, P_{1}, P_{2}, \ldots, P_{n}, \ldots\right)$ on the basis of the state probability $P_{n}$. Then, the entropy $H(\vec{P})$ of the stochastic system is given by

$$
H(\vec{P}) \equiv-\sum_{n=0}^{\infty} P_{n} \ln P_{n} .
$$

Further, the force of changing the entropy of the system is called "the entropic force" in the concept of the statistical mechanics. The entropic force can be defined as $\partial H(\vec{P}) / \partial \vec{P}$ in response to the change of the state probability distribution of the system.

On the other hand, the potential energy $U(\vec{P})$ is defined as

$$
U(\vec{P}) \equiv \sum_{n=0}^{\infty} g(n) P_{n}
$$

where $g(n)$ describes a potential function defined by state $n$ of the system. The absolute value of $g(n)$ means the magnitude of the force, and the sign of $g(n)$ indicates the direction of the force. Since the potential function $g(n)$ is evaluated based on the waiting queue length $n$ in the $M / M / 1$ queueing system, it would be natural that the potential $g(n)$ in the case of $n=0$ is zero. In addition, it can be assumed that the potential $g(1)$ in the case of $n=1$ is rationally defined as $g(1)=1$ as a reference value, that is, a unit value, of the potential function. The force of changing the potential energy $U(\vec{P})$ is called "the energy force". The energy force can be defined as $\partial U(\vec{P}) / \partial \vec{P}$ in response to the change of the state probability distribution of the system.

Based on the concept of the statistical mechanics, the system is led to the steady-state by the balance between the entropic force and the energy force. Then, the steady-state probability distribution of the system can be derived from the relationship of the balance between the entropic force and the energy force as follows:

$$
\frac{\partial}{\partial \vec{P}} H(\vec{P})=\omega_{1} \frac{\partial}{\partial \vec{P}} U(\vec{P})
$$


where $\omega_{1}$ is introduced as an unknown coefficient to unify the scales of the entropic force and the energy force. And then, it is obvious that the following equation should be satisfied:

$$
\sum_{n=0}^{\infty} P_{n}=1
$$

Therefore, based on the concept of the statistical mechanics, we propose the model for analyzing the steadystate probability distribution of the $M / M / 1$ queueing system with balking under the relation of equation (2.3) and the constraint of equation (2.4) as follows:

$$
\Lambda\left(\vec{P}, \omega_{1}, \omega_{2}\right)=\frac{\partial}{\partial \vec{P}}\left\{H(\vec{P})-\omega_{1} U(\vec{P})-\omega_{2}\left(\sum_{n=0}^{\infty} P_{n}-1\right)\right\}=\overrightarrow{0} .
$$

where $\omega_{2}$ mean the Lagrange multiplier. This is analogous to the method of the equilibrium statistical physics where the (Gibbs) free energy is minimized. See Greiner et al. [11] and Preston [29].

As mentioned before, $\omega_{1}$ is introduced as an unknown coefficient to unify the scales of the entropic force and the energy force. Then, the unknown coefficient $\omega_{1}$ is also operated in the same way as the Lagrange multiplier $\omega_{2}$ in the proposed analysis model in equation (2.5). Accordingly, equation (2.5) is understood as a first-order condition regarding the Lagrange function.

As consequence, based on the proposed model in equation (2.5), we derive

$$
P_{n}=\rho^{g(n)} P_{0} .
$$

The detail of the derivation process of equation (2.6) is presented in Appendix A. Furthermore, by comparing equation (2.6) with equation (1.3), we can derive analytically the following formula of arrival rate $\lambda_{n}, \forall n$ :

$$
\lambda_{n}=\lambda \rho^{g(n+1)-g(n)-1} .
$$

See Appendix B for the derivation process of equation (2.7). Hence, by introducing an appropriate potential function $g(n)$, we can derive arrival rate $\lambda_{n}, \forall n$. We will construct the potential function in order that the directivity strength of balking can be represented in the extended analysis model. So, let us consider the required property of the potential function $g(n)$.

Since the relation of $\lambda_{n} \geq \lambda_{n+1}$ is assumed under balking, the following inequality for the potential function should be satisfied in the case of $\rho<1$ :

$$
g(n+1)-g(n)-1 \geq 0, \forall n .
$$

If $g(n)=n$, equation (2.8) is satisfied. Further, equation (2.6) is reduced to equation (1.4). At the same time, from equation $(2.7)$, we have

$$
\lambda_{n}=\lambda .
$$

On the other hand, in the case of $\rho>1$, the potential function should satisfy the following inequality under condition that the steady-state probability distribution exists:

$$
g(n+1)-g(n)-1 \leq 0, \forall n .
$$

It is difficult to define uniquely the potential function $g(n)$ in theory. Therefore, in order that the extended analysis model in this study can reproduce the steady-state probability distribution of equation (1.6) under the situation that the arrival rates are typically assumed as equation (1.5), the potential function $g(n)$ can be obtained as follows:

$$
g(n)=n-\frac{\ln n !}{\ln \rho}
$$


The detail of the derivation process of equation (2.11) is presented in Appendix C. Furthermore, in order to represent the directivity strength of balking, for convenience, we would provide the potential function $g(n)$ as follows:

$$
g(n)=n-\frac{r \ln n !}{\ln \rho},
$$

where $r(\geq 0)$ denotes the parameter of adjusting the directivity strength of balking, and the case of $r=0$ means the situation without balking. It can be found that the potential function $g(n)$ includes equation $(2.11)$ as a special case of $r=1$ and $g(n)=n$ giving equation (2.9) as a special case of $r=0$, and satisfies some reasonable requirements mentioned above.

By applying equation (2.12), we can obtain as follows:

$$
\begin{aligned}
& P_{n}=\rho^{n-\frac{r \ln n !}{\ln \rho}} P_{0}=\rho^{n} e^{-r \ln n !} P_{0}=\frac{\rho^{n} P_{0}}{(n !)^{r}}, \\
& \frac{1}{P_{0}}=\sum_{n=0}^{\infty} \frac{\rho^{n}}{(n !)^{r}}, \\
& \lambda_{n}=\lambda \rho^{-\frac{r \ln (n+1)}{\ln \rho}}=\lambda e^{-r \ln (n+1)}=\frac{\lambda}{(n+1)^{r}} .
\end{aligned}
$$

Note that equations (2.13)-(2.15) are also approved under $\rho=1$.

In the case of $r=0$, it is obvious that equation (2.15) is reduced to the the constant arrival rate $\lambda$ in the $M / M / 1$ queueing system without balking, and then, equations (2.13) and (2.14) give the the steady-state probability distribution of equation (1.4) in the $M / M / 1$ queueing system without balking. On the other hand, it is easily found that equations (1.5) and (1.6) in the $M / M / 1$ queueing system with balking can be reproduced through equations (2.13)-(2.15) under $r=1$. From the above discussion, we have found that the potential function of equation (2.12) can reproduce the steady-state probability distributions in equations (1.4) and (1.6) as each special case. Further, since we can see from equation (2.15) that $\lambda_{n}$ decreases due to increase of $r$, it is obvious that the influence of balking becomes strong with the increase of $r$. Therefore, the parameter $r$ can adjust the directivity strength of balking. As consequence, it can be found that the extended analysis model formulated by the relationship of equation (2.5) based on the concept of the statistical mechanics includes the traditional $M / M / 1$ queueing model without balking and the $M / M / 1$ queueing model with balking considered by Abou-El-Ata and Hariri [1], El-Sherbiny [9], Natvig [25], and Sztrik [35].

\section{NumericAl VERIFICATIONS}

Figure 1 illustrates the relations between the arrival rate $\lambda_{n}$ and the waiting queue length derived from equation (2.15) under the condition of $\lambda=20$ and $\mu=25$ as an example. In addition, the difference in the arrival rate $\lambda_{n}$ by the parameter $r$ is shown in Figure 1. Since the relationship of $\lambda_{n} \geq \lambda_{n+1}$ is satisfied in Figure 1 for any $r$, it is found that balking considered in this study has been successfully explained. From equation (2.15), it is obvious that the arrival rates $\lambda_{n}, \forall n$ are a constant as $\lambda_{n}=\lambda$ in the case of $r=0$. Furthermore, when the parameter $r$ becomes larger, the arrival rate $\lambda_{n}$ decreases faster. This fact means that the influence of balking becomes stronger in the case that the parameter $r$ is larger. That is, it is seen that the potential function in equation (2.12) could represent the influence of the directivity strength of balking.

Next, the steady-state probability distributions under various values of the parameter $r$ are illustrated in Figure 2 under the condition of $\lambda=20$ and $\mu=25$ as an example. From the results in Figure 2, the larger the parameter $r$ is, the more sharp the configuration of the steady-state probability distribution against the waiting queue length $n$ is. This feature also implies that the increase of the parameter $r$ makes the influence of balking stronger. 


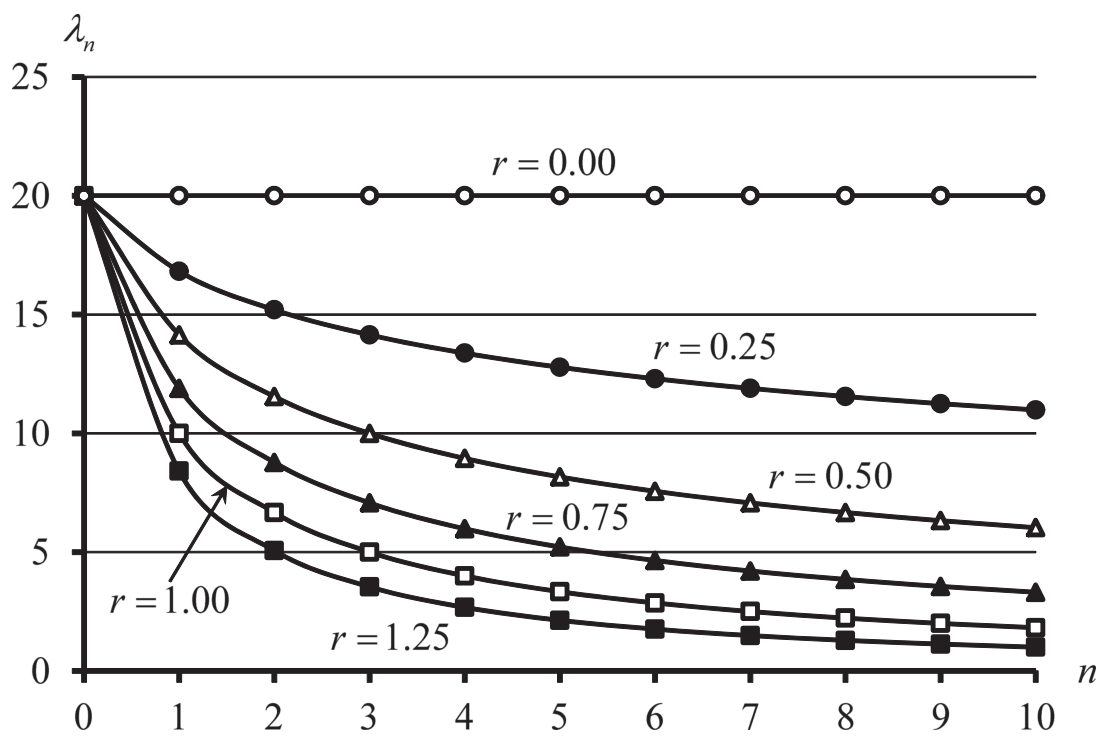

FiguRE 1. The relationship between the waiting queue length $n$ and the arrival rate $\lambda_{n}$ for each $r$ in the case of $\mu=25$ and $\lambda=20$.

Furthermore, a numerical example in the case of $\rho>1$ is investigated. Figures 3 and 4 show the arrival rates and the steady-state probability distributions under the condition of $\lambda=30, \mu=25$ and respective values of the parameter $r$. From the results in Figures 3 and 4, it is found that the extended analysis model based on the concept of the statistical mechanics has derived the arrival rates and the steady-state probability distributions in the situation of $\rho>1$ appropriately. In addition, we can see the influence of the parameter $r$ similar to the results in Figures 1 and 2.

Through Figures 1-4, the influence and effect of the directivity strength of balking are understood in both situations of $\rho<1$ and $\rho>1$. Therefore, the usefulness of the extended analysis model constructed based on the concept of the statistical mechanics is found.

The potential function $g(n)$ is defined by equation (2.12) for convenience. That is, it might be not the one and only one. However, we can investigate some features in the $M / M / 1$ queueing system with balking based on the extended analysis model including the potential function $g(n)$. Then, if the directivity strength of balking can be known, we can consider the action for improving the queueing system performance. In next section, we consider the procedure for estimating the directivity strength of balking based on the extended analysis model constructed based on the concept of the statistical mechanics. Some numerical results are shown. That is, it will be found that the potential function $g(n)$ is practically useful, even though it was decided for convenience.

\section{Estimation of ARRIVAL RATES BY UtiLizing EXTENDED ANALYSiS MODEL}

As mentioned before, in the traditional analysis of the $M / M / 1$ queueing system under the consideration of balking, the steady-state probability distribution has been derived under the assumption that all arrival rates in each waiting queue length can be known. However, it does not necessarily guarantee that arrival rates for every waiting queue length are justifiably known. Furthermore, it is actually hard to estimate the arrival rates $\lambda_{n}$ for every waiting queue length even though observing the $M / M / 1$ queueing system with balking. In addition, there should be various situations for the directivity strength of balking in practice.

Hence, we consider the procedure for estimating arrival rates for respective waiting queue lengths based on the extended analysis model constructed based on the concept of the statistical mechanics. Remark that the 


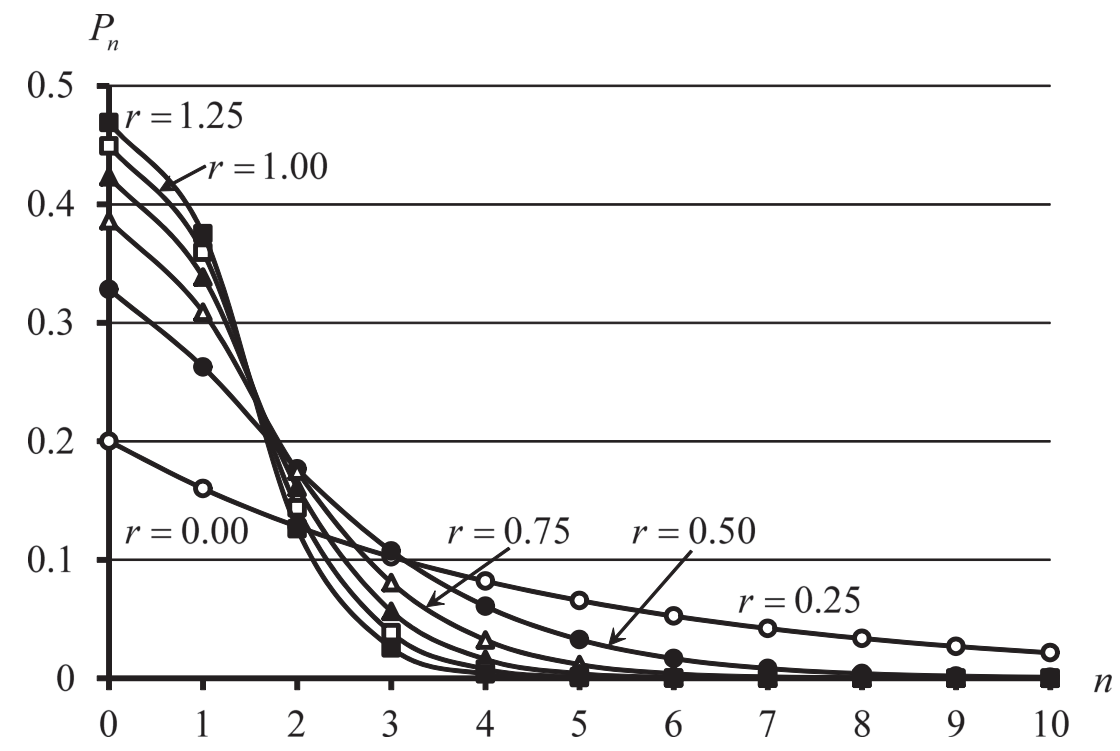

Figure 2. The relationship between the waiting queue length $n$ and the steady-state probability $P_{n}$ in the case of $\mu=25$ and $\lambda=20$.

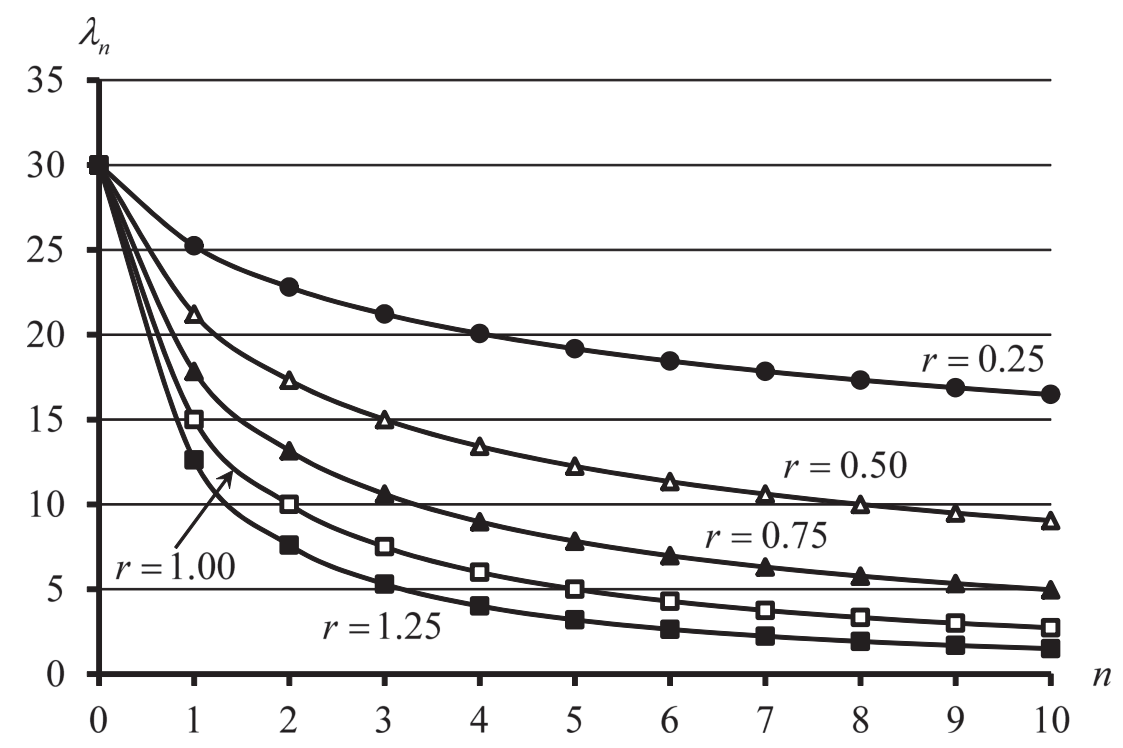

FiguRE 3 . The relationship between the waiting queue length $n$ and the arrival rate $\lambda_{n}$ for each $r$ in the case of $\mu=25$ and $\lambda=30$. 


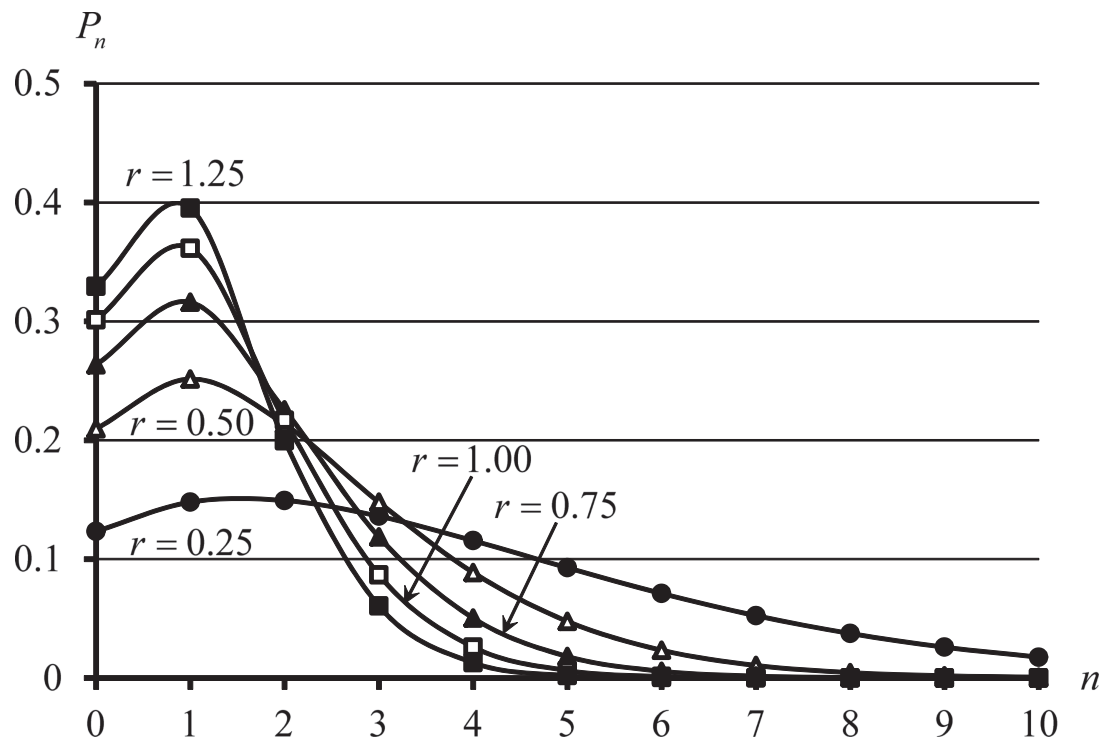

FIGURE 4 . The relationship between the waiting queue length $n$ and the steady-state probability $P_{n}$ in the case of $\mu=25$ and $\lambda=30$.

steady-state probability distribution can be derived by prescribing only the potential function $g(n)$ defined conveniently. In the case of specifying the potential function $g(n)$ as equation (2.12), it is required only to obtain the parameter $r$. Therefore, in such a purpose, it is sufficient to estimate the directivity strength of balking in the extended analysis model constructed based on the concept of the statistical mechanics. That is, the parameter $r$ is a key parameter for clarifying the internal structure of the queuing system. Therefore, based on the extended analysis model in this study, we discuss the procedure for estimating the parameter $r$ by using observations from the $M / M / 1$ queueing system.

Assume that the frequencies for each waiting queue length are observed through the repeated observation for the $M / M / 1$ queueing system. Based on this observation, we can estimate the observed state probability $P_{n}^{\ddagger}$. As a result, we can employ the concept of the goodness of fit test based on the chi-square statistic for the purpose of determining optimally the parameter $r$ as $r \equiv r^{*}$. Based on the chi-square statistic under the goodness of fit test, we estimate the optimal $r^{*}$ to minimize the following evaluation:

$$
J=\sum_{n=0}^{K} \frac{\left(P_{n}^{\ddagger}-P_{n}^{(r)}\right)^{2}}{P_{n}^{(r)}},
$$

where $P_{n}^{\ddagger}$ denotes the observed state probability and $P_{n}^{(r)}$ denotes the theoretical steady-state probability under the parameter $r$ based on equation (2.13). In addition, $K$ means the number of states appropriate for estimation, where $K$ is given from the result that some states of small probability $P_{n}^{(r)}, n>K-1$ are unified. Note that we can obtain the observed state probability $P_{n}^{\ddagger}$ based on the observation of the frequency about waiting queue length $n$ through the repeated observation. Then, we can find the optimal $r^{*}$ that minimizes $J$ by searching for any $r$. Therefore, the extended analysis model based on the concept of the statistical mechanics can be recognized as a useful model that can reproduce the system based on the data through the simple observation.

Through the Monte Carlo simulation, the validity of the estimation procedure described above is confirmed. Here, as a numerical example, we assume the parameter of adjusting the directivity strength of balking as $r=0.25$, where note that the value of $r$ is unknown. In addition, suppose $\lambda=30$ and $\mu=25$. Then, we 
TABLE 1. One numerical example about the state probability distribution in the case of $r=0.25$ and $n=300$.

\begin{tabular}{llll}
\hline$n$ & $P_{n}^{\dagger}$ & $P_{n}^{\ddagger}$ & $P_{n}^{\left(r^{*}\right)}$ \\
\hline 0 & 0.123284 & 0.110000 & 0.118617 \\
1 & 0.147940 & 0.140000 & 0.142340 \\
2 & 0.149283 & 0.136667 & 0.143632 \\
3 & 0.136117 & 0.140000 & 0.131875 \\
4 & 0.115499 & 0.110000 & 0.113136 \\
5 & 0.092687 & 0.113333 & 0.092058 \\
6 & 0.071066 & 0.076667 & 0.071729 \\
7 & 0.164125 & 0.173333 & 0.186613 \\
\hline
\end{tabular}

TABLE 2. Estimated result of $r^{*}$ through 100 trials.

\begin{tabular}{lllll}
\hline 0.24 & 0.27 & 0.26 & 0.26 & 0.27 \\
0.24 & 0.25 & 0.24 & 0.25 & 0.26 \\
0.24 & 0.27 & 0.25 & 0.26 & 0.24 \\
0.25 & 0.24 & 0.24 & 0.27 & 0.25 \\
0.27 & 0.24 & 0.25 & 0.26 & 0.26 \\
0.24 & 0.27 & 0.25 & 0.26 & 0.27 \\
0.28 & 0.27 & 0.24 & 0.28 & 0.25 \\
0.26 & 0.26 & 0.25 & 0.24 & 0.23 \\
0.25 & 0.25 & 0.28 & 0.28 & 0.27 \\
0.27 & 0.23 & 0.24 & 0.25 & 0.25 \\
0.23 & 0.28 & 0.25 & 0.26 & 0.24 \\
0.25 & 0.25 & 0.25 & 0.24 & 0.23 \\
0.25 & 0.22 & 0.27 & 0.24 & 0.27 \\
0.23 & 0.27 & 0.27 & 0.25 & 0.25 \\
0.26 & 0.26 & 0.25 & 0.26 & 0.25 \\
0.23 & 0.26 & 0.24 & 0.25 & 0.25 \\
0.26 & 0.26 & 0.25 & 0.24 & 0.24 \\
0.26 & 0.22 & 0.25 & 0.25 & 0.25 \\
0.27 & 0.24 & 0.25 & 0.24 & 0.25 \\
0.25 & 0.27 & 0.25 & 0.26 & 0.24 \\
\hline
\end{tabular}

generate some random numbers under the steady-state probability distribution in equation (2.13). Based on the estimation procedure constructed in this section, the value of $r$ is estimated as $r^{*}$.

In Table 1, the probability distribution $P_{n}^{\dagger}$ indicates the theoretical steady-state probability distribution under $r=0.25$. The probability distribution $P_{n}^{\ddagger}$ in Table 1 shows a numerical example about the state probability distribution based on 300 observation data, that is, the state probability distribution based on the simulation. Then, all states where the theoretical steady-state possibility is less than 0.05 are combined into one state. Further, the probability distribution $P_{n}^{\left(r^{*}\right)}$ presents the reproduced steady-state probability distribution under the estimated parameter $r^{*}$. In the case of Table 1, we have obtained the estimated value $r^{*}=0.24$. From the result in Table 1, it is considered that the state probability distribution of $P_{n}^{\left(r^{*}\right)}$ have reproduced precisely the steady-state probability distribution $P_{n}^{\dagger}$ in the simulation.

Next, in order to confirm the estimated precision of $r^{*}$, we have conducted the numerical analysis of 100 iterations under same condition. We show the estimated results of $r^{*}$ in Table 2. From Table 2, we have evaluated the average and standard deviation of estimated results of $r^{*}$. The average and standard deviation 
TABLE 3. Basic statistics in this numerical example.

\begin{tabular}{ll}
\hline Average & Standard deviation \\
\hline 0.2526 & 0.013537 \\
\hline
\end{tabular}

have been shown in Table 3. Then, we know that the estimator $r^{*}$ have enough precision. It has been confirmed that similar results have been obtained under different conditions.

The potential function $g(n)$ with the parameter $r$ of the directivity strength of balking might not be a unique potential function derived in theory. However, when we accept this formula conceptually as a model function, the value of the directivity strength of balking can be estimated based on observations from the $M / M / 1$ queueing system. Thereafter, we can consider the action for improving the queueing system performance. That is, it should be found that the potential function $g(n)$ is practically useful.

\section{Concluding Remarks}

In the traditional steady-state analysis of the $M / M / 1$ queueing system with balking, all arrival rates for any waiting queue length have been provided in advance. Although it has been typically assumed in the traditional studies that the arrival rates obey an inverse proportional function for the waiting queue length, the relation between the waiting queue length and the degree of occurrence of balking, that is, the diversity of the directivity strength of balking has not been considered. In this study, we have proposed the extended analysis model for deriving the steady-state probability distribution of the $M / M / 1$ queueing system with balking based on the concept of the statistical mechanics. As a result, the steady-state probability and the arrival rates for each waiting queue length under the consideration of the influence of the directivity strength of balking have been formulated.

The results based on the proposed analysis model for the $M / M / 1$ queueing system with balking have included the traditional result for the $M / M / 1$ queueing system without balking based on the theory of the stochastic process, and the typical result of the $M / M / 1$ queueing system with balking under the situation that the arrival rates are assumed to equation (1.5) as a special case of $r=1$. Further, the extended analysis model presented in this study can adjust the directivity strength of balking by using the parameter $r$. Accordingly, we can understand that the extended analysis model based on the concept of the statistical mechanics is an analytical model with the versatility. Furthermore, it has been shown that the extended analysis model can derive the arrival rates $\lambda_{n}$ as a function under the consideration of the directivity strength of balking. Then, the procedure for estimating the parameter $r$ for the directivity strength of balking in the extended analysis model has been successfully constructed. As the result, by utilizing the extended analysis model, we can estimate the arrival rates $\lambda_{n}$ through the observation result of the $M / M / 1$ queueing system.

Further, we have constructed the procedure for estimating the arrival rates through the observation result about the frequencies of respective waiting queue lengths in the $M / M / 1$ queueing system based on the extended analysis model. As the result, the extended analysis model for deriving the steady-state probability distribution and the arrival rates for each waiting queue length of the $M / M / 1$ queueing system with balking has been successfully proposed based on the concept of the statistical mechanics.

In this consideration, the potential function has been conveniently provided. That is, it might be not the one and only one. However, although it is not the one and only one, the potential function in this study is useful and effective to analyze the steady-state probability distribution of the $M / M / 1$ queueing system with balking and to evaluate the directivity strength of balking.

Then, in the case of considering the action for improving the queueing system performance based on the estimate of the directivity strength of balking, improvement of service rate is conceivable at first. In addition, the steady-state analysis for the $M / M / c$ queueing system with balking would be also considered. In such a situation, a model for analyzing the steady-state probability distribution of the $M / M / c$ queueing system with 
balking should be required. The further extension to analyze the steady-state probability distribution of the $M / M / c$ queueing system with balking is also considered as an interesting problem. We would like to deal with this issue as an immediate challenge.

\section{Appendix A. Derivation of equation (2.6)}

From equation (2.5), we have

$$
\begin{aligned}
\frac{\partial}{\partial P_{n}}\left\{-\sum_{n=0}^{\infty} P_{n} \ln P_{n}-\omega_{1} \sum_{n=0}^{\infty} g(n) P_{n}-\omega_{2}\left(\sum_{n=0}^{\infty} P_{n}-1\right)\right\} \\
=-\ln P_{n}-1-\omega_{1} g(n)-\omega_{2} \\
=0, \quad \forall n \geq 0 .
\end{aligned}
$$

Hence, the following formula is obtained:

$$
P_{n}=\alpha \beta^{g(n)},
$$

where $\alpha=e^{-1-\omega_{2}}$ and $\beta=e^{-\omega_{1}}$. Besides, by substituting the equilibrium state probability of equation (A.2) into equation (2.4), $\alpha$ can be written as

$$
\alpha=\frac{1}{\sum_{n=0}^{\infty} \beta^{g(n)}} .
$$

Then, we can derive

$$
P_{n}=\frac{\beta^{g(n)}}{\sum_{k=0}^{\infty} \beta^{g(k)}} .
$$

Moreover, since $g(0)=0$ and $g(1)=1$, we obtain

$$
\begin{aligned}
& P_{0}=\frac{1}{\sum_{k=0}^{\infty} \beta^{g(k)}}, \\
& P_{1}=\frac{\beta}{\sum_{k=0}^{\infty} \beta^{g(k)}} .
\end{aligned}
$$

From equations (A.6) and (A.6), we have the relationship of

$$
P_{1}=\beta P_{0} .
$$

On the other hand, from equation (1.1), the relationship of $\lambda P_{0}-\mu P_{1}=0$ is also satisfied in the $M / M / 1$ queueing system with balking. Therefore, we obtain

$$
\beta=\rho .
$$

Consequently, it is possible to give the equilibrium state probability distribution of the $M / M / 1$ queueing system with balking as follows:

$$
P_{n}=\frac{\rho^{g(n)}}{\sum_{k=0}^{\infty} \rho^{g(k)}} .
$$


Further, since $P_{0}$ is represented as

$$
P_{0}=\frac{1}{\sum_{k=0}^{\infty} \rho^{g(k)}}
$$

$P_{n}$ for any $n$ are described finally as

$$
P_{n}=\rho^{g(n)} P_{0}
$$

\section{Appendix B. Derivation of Equation (2.7)}

Based on equations (1.3) and (2.6), we have

$$
\frac{P_{n+1}}{P_{n}}=\frac{\lambda_{n}}{\mu}=\rho^{g(n+1)-g(n)} .
$$

Therefore, we have

$$
\lambda_{n}=\mu \rho^{g(n+1)-g(n)}=\lambda \rho^{-1} \rho^{g(n+1)-g(n)}=\lambda \rho^{g(n+1)-g(n)-1} .
$$

\section{Appendix C. Derivation of equation (2.11)}

Based on equation (2.7), the formula of arrival rate $\lambda_{k}, \forall k$ can be generally presented as

$$
\lambda_{k}=f(k ; \lambda) .
$$

Accordingly, we have

$$
-\ln \lambda+\ln f(k ; \lambda)=\{g(k+1)-g(k)-1\} \ln \rho .
$$

By summing equation (C.2) from $k=0$ to $k=n$, we can obtain as follows:

$$
-(n+1) \ln \lambda+\sum_{k=0}^{n} \ln f(k ; \lambda)=\{g(n+1)-(n+1)\} \ln \rho,
$$

where remark $g(0)=0$ and $g(1)=1$. From equation (C.3), the potential function $g(n)$ can be generally described as follows:

$$
g(n)=n-\frac{n \ln \lambda}{\ln \rho}+\frac{\sum_{k=0}^{n-1} \ln f(k ; \lambda)}{\ln \rho} .
$$

By assuming typically $f(k ; \lambda)$, that is, the arrival rates $\lambda_{k}$, as equation $(1.5)$, the potential function $g(n)$ can be obtained as follows:

$$
\begin{aligned}
g(n) & =n-\frac{n \ln \lambda}{\ln \rho}+\frac{\sum_{k=0}^{n-1} \ln \frac{\lambda}{k+1}}{\ln \rho} \\
& =n-\frac{n \ln \lambda}{\ln \rho}+\frac{n \ln \lambda-\ln n !}{\ln \rho} \\
& =n-\frac{\ln n !}{\ln \rho} .
\end{aligned}
$$


Additionally, when $f(k ; \lambda)=\lambda_{k}$ is assumed as

$$
f(k ; \lambda)=\lambda_{k}=\frac{\lambda}{(k+1)^{r}}
$$

we can derive

$$
\begin{aligned}
g(n) & =n-\frac{n \ln \lambda}{\ln \rho}+\frac{\sum_{k=0}^{n-1} \ln \frac{\lambda}{(k+1)^{r}}}{\ln \rho} \\
& =n-\frac{n \ln \lambda}{\ln \rho}+\frac{n \ln \lambda-r \ln n !}{\ln \rho} \\
& =n-\frac{r \ln n !}{\ln \rho} .
\end{aligned}
$$

Acknowledgements. This work was supported by Japan Society for the Promotion of Science (JSPS) KAKENHI Grant Number 18K04611: "Evaluation of system performance and reliability under incomplete information environment".

\section{REFERENCES}

[1] M.O. Abou-El-Ata and A.M.A. Hariri, The M/M/c/N queue with balking and reneging. Comput. Oper. Res. 19 (1992) $713-716$.

[2] I. Arizono, Y. Cui and H. Ohta, An analysis of M/M/s queueing systems based on the maximum entropy principle. J. Oper. Res. Soc. 42 (1991) 69-73.

[3] G.R.M. Borzadaran, A note on maximum entropy in queueing problems. Econ. Qual. Control 24 (2009) $263-267$.

[4] D. Chandler, Introduction to Modern Statistical Mechanics. Oxford University Press (1987).

[5] C.Chen, Z. Jia and P. Varaiya, Causes and cures of highway congestion. IEEE Control Syst. 21 (2001) $26-32$.

[6] F.R.B. Cruz, J. MacGregor Smith and D.C. Queiroz, Service and capacity allocation in M/G/c/c state-dependent queueing networks. Comput. Oper. Res. 32 (2005) 1545-1563.

[7] F.R.B. Cruz and J. MacGregor Smith, Approximate analysis of M/G/c/c state-dependent queueing networks. Comput. Oper. Res. 34 (2007) 2332-2344.

[8] D. Dickson, R.C. Ford and B. Laval, Managing real and virtual waits in hospitality and service organizations. Cornell Hosp. Q. 46 (2005) 52-68.

[9] A.A. El-Sherbiny, The truncated heterogeneous two-server queue: $\mathrm{M} / \mathrm{M} / 2 / \mathrm{N}$ with reneging and general balk function. Int. J. Math. Arch. 3 (2012) 2745-2754.

[10] A. Economou and S. Kanta, Optimal balking strategies and pricing for the single server Markovian queue with compartmented waiting space. Queueing Syst. 59 (2008) 237-269.

[11] W. Greiner, L. Neise and H. Stöcker, Thermodynamics and Statistical Mechanics. Springer-Verlag, New York (1995).

[12] S. Guiasu, Maximum entropy condition in queueing theory. J. Oper. Res. Soc. 37 (1986) 293-301.

[13] R. Hassin and M. Haviv, To Queue or Not to Queue. Springer-Verlag, New York (2003).

[14] R. Hassin, Rational Queueing. CRC press (2016).

[15] M. Haviv and Y. Kerner, On balking from an empty queue. Queueing Syst. 55 (2007) 239-249.

[16] M. Jain and M.R. Dhakad, Maximum entropy analysis for G/G/1 queuing system. Int. J. Eng. Trans. A: Basics 16 (2003) 163-170.

[17] N.K. Jain, R. Kumar and B. Kumar Som, An M/M/1/N queuing system with reverse balking. Am. J. Oper. Res. 4 (2014) 17-20.

[18] B.K. Kumar, P.R. Parthasarathy and M. Sharafali, Transient solution of an M/M/1 queue with balking. Queueing Syst. 13 (1993) 441-448.

[19] D. Kouvatsos and N. Tabet-Aouel, An ME-based approximation for multi-server queues with preemptive priority. Eur. J. Oper. Res. 77 (1994) 496-515.

[20] D.D. Kouvatsos, J.S. Alanazi, and K. Smith, A unified ME algorithm for arbitrary open QNMs with mixed blocking mechanisms. Numer. Algebra Control Optim. (NACO) 1 (2011) 781-816.

[21] J. Li and L. Liu, On an M/G/1 queue in random environment with Min(N, V) policy. RAIRO: OR 52 (2018) 61-77.

[22] L. Liu and V.G. Kulkarni, Explicit solutions for the steady state distributions in M/PH/1 queues with workload dependent balking. Queueing Syst. 52 (2006) 251-260.

[23] L. Liu and V.G. Kulkarni, Busy period analysis for M/PH/1 queues with workload dependent balking. Queueing Syst. 59 (2008) 37-51. 
[24] A. Montazer-Haghighi, J. Medhi and S.G. Mohanty, On a multiserver markovian queueing system with balking and reneging. Comput. Oper. Res. 13 (1986) 421-425.

[25] B. Natvig, On the transient state probabilities for a queueing model where potential customers are discouraged by queue length. J. Appl. Prob. 11 (1974) 345-354.

[26] B. Natvig, On a queuing model where potential customers are discouraged by queue length. Scand. J. Stat. 2 (1975) 34-42.

[27] R.P. Nithya and M. Haridass, Analysis of a queueing system with two phases of bulk service, closedown and vacation interruption. Int. J. Appl. Eng. Res. 11 (2016) 467-468.

[28] B. Prabhakar, Entropy and the timing capacity of discrete queues. IEEE Trans. Inf. Theor. 49 (2003) $357-370$.

[29] C. Preston, Gibbs States on Countable Sets. Cambridge University Press, London (1974).

[30] P. Rajadurai, Sensitivity analysis of an $M / G / 1$ retrial queueing system with disaster under working vacations and working breakdowns. RAIRO: OR 52 (2018) 35-54.

[31] G. Rubin and D.S. Robson, A single server queue with random arrivals and balking: Confidence interval estimation. Queueing Syst. 7 (1990) 283-306.

[32] C.J. Singh, M. Jain and B. Kumar, Analysis of $\mathrm{M}^{\mathrm{X}} / \mathrm{G} / 1$ queueing model with balking and vacation. Int. J. Oper. Res. 19 (2014) 154-173.

[33] S.N. Singh and S.B. Tiwari, An application of generalized entropy in queueing theory. J. Appl. Sci. Eng. 16 (2013) 99-103.

[34] R. Sudhesh, A. Azhagappan and S. Dharmaraja, Transient analysis of M/M/1 queue with working vacation, heterogeneous service and customers' impatience. RAIRO: OR 51 (2017) 591-606.

[35] J. Sztrik, Basic Queueing Theory. Faculty of Informatics, University of Debrecen, Hungary (2012).

[36] M. Toda, R. Kubo and N. Saito, Statistical Physics I. Springer, Berlin (1983).

[37] K.-H. Wang, S.-L. Chuang and W.-L. Pearn, Maximum entropy analysis to the N policy M/G/1 queueing system with a removable server. Appl. Math. Model. 26 (2002) 1151-1162.

[38] A.R. Ward and P.W. Glynn, A diffusion approximation for a GI/GI/1 queue with balking or reneging. Queueing Syst. 50 (2005) 371-400. 